\title{
Geometric optimisation of chicanes using driving simulator trajectory data
}

1 Kadir Akgol MSc, PhD

Lecturer, Department of Transportation, Giresun University, Giresun, Turkey (corresponding author: kadirakgol@hotmail.com.tr) (Orcid:0000-0002-1939-6717)

2 Banihan Gunay MSc, PhD Professor, Freelance Academic, Muratpasa, Antalya, Turkey (Orcid:0000-0002-4640-417X)
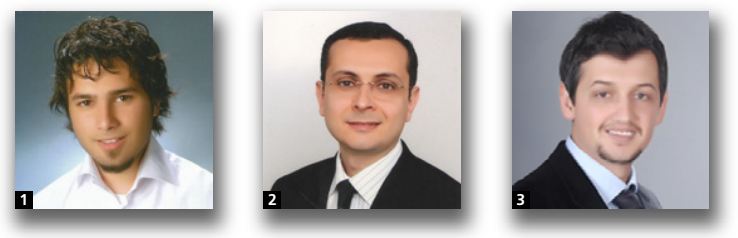

Vertical traffic-calming applications may possess certain disadvantages. For instance, drivers sometimes apply a harsh brake very late or they may even prefer to accelerate rather than slow down to by-pass the suspension's shock threshold level. This has negative consequences on both vehicular and pedestrian traffic. In this study, the effects of chicanes - one of the most common horizontal speed reduction applications - on driver behaviour were examined and certain design characteristics were evaluated in the traffic laboratory of Akdeniz University in Turkey. To complete the driving tests safely and in an economical fashion, they were performed in a driving simulator. The trajectory data of some 125 drivers were saved by the simulator software. To examine these data in detail, a new method was developed using VisualLisp programming. The trajectory data were then transferred to an ArcGIS analysis program and the driving test data on the virtual chicanes were evaluated according to position and speed data. Finally, the optimum chicane design characteristics and their efficiency were determined.

\section{Notation}

$\operatorname{Left}\left(x_{i}, y_{i}\right)$

$m$

$O\left(x_{i}, y_{i}\right) \quad$ coordinates for the centreline

p calculated probability

$\operatorname{Right}\left(x_{i}, y_{i}\right) \quad$ coordinates of right wheel

$x_{i} \quad$ coordinate $x$ of $i$ th point

$y_{i} \quad$ coordinate $y$ of $i$ th point

$\Delta x \quad$ amount of shift in $x$-direction

$\Delta y \quad$ amount of shift in $y$-direction

\section{Introduction}

Previous studies have shown that one of the most complained about transport problems within settlement areas is the high speeds of vehicles (Bahar, 2007; Peterson, 2006). In general, vertical traffic-calming applications (e.g. speed cushions, speed bumps and rumble strips) are considered to be the default solution in various problematic locations. However, drivers usually only realise the presence of these very late when approaching at high speeds and thus apply brakes harshly or drive over them without reducing speed. Additionally, some drivers even speed up after speed bumps to compensate for the speed drop. It is important to select and design the most suitable type of traffic-calming instrument with different characteristics, as each application will have unique design characteristics and aims.

In a study by Macbeth (1998), the width of an examined road was reduced from $8.4 \mathrm{~m}$ to $6.4 \mathrm{~m}$ by using central islands as chicanes, and it was observed that the 85 th percentile speed decreased from $49.8 \mathrm{~km} / \mathrm{h}$ to $44.8 \mathrm{~km} / \mathrm{h}$. In a similar study Corkle et al. (2001) calculated that, upon the application of chicanes, the 85 th percentile speed value decreased by about $6 \%$. In another study, Sayer et al. (1998) observed that chicanes had a greater effect than speed bumps or cushions on the reduction of vehicle speeds. Accidents involving injuries have been reported to have decreased by $54 \%$ on urban roads where chicanes have been applied (DfT, 2006).

Any traffic proposal should be designed with extreme care before being put into use. Where possible, they should be tested to see the outcomes before being put into practice. This can be achieved by means of simulation software or simulators created in the computer medium, which reflect reality as far as possible. For example, the driving trajectories produced by simulators make it possible to examine the effects of various design features on driver behaviour. However, using currently available techniques, it is impossible to examine vehicular movements in microscopic detail. 
In this study, a new method was developed to examine vehicular movements in detail using special commercial software. By employing this method, the design characteristics of different chicanes were optimised. For this purpose, 12 different chicane designs were developed as they are one of the least known traffic-calming applications in the country where this study was conducted (Turkey). The effect of the chicanes on the lateral positions and speeds of vehicles were studied in a simulator environment. The tests conducted involved 125 volunteer drivers. The position and speed data of each driver were recorded individually and all movements of the vehicle on the virtual test road were recorded. Vehicular movements can be monitored by drawing an $x-y$ scatter plot for position data. However, with this method it is quite difficult to see exactly where a vehicle is located on the roadway, laterally and longitudinally. When all driver movements are superimposed, the graph becomes even more chaotic. Therefore, in this study, the data obtained were transferred to ArcGIS software in order to examine the position and speed data of vehicles in detail and with excellent clarity. This produced conclusive findings on the efficiency of various chicane applications with various geometric characteristics.

In transportation, simulators are predominantly used in aviation and navigation, but their application in the field of highways is on the increase. It is possible to divide simulator applications for highways into two main groups - those for educational purposes and those for research purposes. In general, the factors affecting driver behaviour and performance have been examined in simulator studies for research purposes. The effects of many different situations on driver behaviour and performance have been studied, including the effects of bicycle riders in rural regions (Duivenvoorden et al., 2015), messaging while driving (Rumschlag et al., 2015), delineators of different configurations (Nygardhs et al., 2014), the use of drugs (Bergeron and Paquette, 2014), roads with and without kerbs (Yang et al., 2014), roads under ice/snow (Hu and Pei, 2012), sadness (Pecher et al., 2012), cruise control (Vollrath et al., 2011), time pressure on left-turns (Gelau et al., 2011), acceleration lanes (Calvi and De Blasiis, 2011), deceleration lanes (Calvi et al., 2012) and variable message signs' traffic information (Lim et al., 2010). Based on analyses of driver behaviour and performance, such studies provide useful information to be taken into consideration when solving trafficrelated problems. Carrying out such research studies without using a simulator would be both costly and risky.

In this study, the simulator software used was able to record the centreline positions of vehicles on the test track. These data were first converted into coordinates to give the right- and lefthand wheel paths and then these coordinates were converted into lines to create the traces of vehicular movement using special commercially available software. By transferring these vehicle tracks to ArcGIS, the zones that were heavily used on the test road were determined using the line density method.
Finally, the position data were converted into point data in ArcGIS and weighted according to the speeds using the kernel density method, and then the effects of the chicanes on the change in speed were examined using ArcGIS software (version 10.3) and the density toolset in the spatial analyst toolbox.

Gunay and Woodward (2007) recorded the lateral position of traffic from a point facing the road by means of a camera and evaluated the lateral distribution of vehicles across the carriageway for various traffic engineering perspectives. Kanagaraj et al. (2015) obtained the trajectory data of vehicles by camera recording and found that there was a statistically significant difference among the lateral position selections of different types of vehicles. Bayer and Hauser (2012) developed an approach for the trajectory optimisation of vehicles and aimed to minimise the passing times of vehicles by means of a chicane within this framework. However, not many comprehensive studies on this subject are available in the literature as it is difficult to draw the trajectories generated for vehicular movements in detail.

VisualLisp programming is used to design intermediate moulds and automatic aspheric lenses for audio products (Lee et al., 2010; Park et al., 2007). It was used by Drăgoi and Navalušić (2013) to collect data in three-dimensional (3D) scanning, by Filip and Neagoe (2010) to imitate the movements of hard-to-understand metal spinning parts and by Kim et al. (2011) to accelerate the multi-stage profile design process and to reduce production costs. In a different approach from previous studies, VisualLisp was used to obtain the trajectories of drivers in simulator tests in this study. Using the VisualLisp programming technique, vehicular movements were easily generated in detail.

\section{Method}

\subsection{Experimental design}

In this study, 12 chicanes with different geometric design features were examined. Six were applied on a two-lane dual carriageway and four were applied on a two-lane single carriageway. In addition, two new chicane design types were developed for testing on dual carriageways. In all the applications, the particular aim was to examine the effective widths at the chicane island and the leading/trailing face angles of these islands. Thus, different designs with effective widths of $2 \cdot 7,3 \cdot 0$, 3.3 and $5.0 \mathrm{~m}$ and leading/trailing face angles of 30,45 and $60^{\circ}$ were created, as detailed in Table 1 . The lane width was $4.0 \mathrm{~m}$ along the entire test road, whereas the length between the start of the stagger on the offside and the end of the stagger on the nearside was $18 \cdot 0 \mathrm{~m}$.

After the geometric characteristics of the chicanes were determined, drawings were then produced in three dimensions. Akdeniz University campus in Turkey was selected as the test road. The overall structure of the test road (topography, 
Table 1. Geometric characteristics of the 12 different chicanes

\begin{tabular}{|c|c|c|c|c|}
\hline Chicane design & Chicane island geometry & Effective lane width: $m$ & Leading/trailing face angle: degrees & Number of islands \\
\hline \multicolumn{5}{|c|}{ Two-lane dual carriageway } \\
\hline 1 & Parabolic & $2 \cdot 7$ & - & 5 \\
\hline 2 & Parabolic & $2 \cdot 7$ & - & - \\
\hline 3 & Cornered & $3 \cdot 0$ & $30 / 30$ & 2 \\
\hline 4 & Cornered & $3 \cdot 0$ & $60 / 30$ & 2 \\
\hline 5 & Cornered & $3 \cdot 0$ & $45 / 45$ & 3 \\
\hline 6 & Cornered & $5 \cdot 0$ & $45 / 45$ & 2 \\
\hline 7 & Cornered & $3 \cdot 0$ & $45 / 45$ & 2 \\
\hline 8 & Parabolic & $3 \cdot 0$ & $45 / 45$ & 2 \\
\hline \multicolumn{5}{|c|}{ Two-lane single carriageway } \\
\hline 9 & Cornered & $3 \cdot 3$ & $60 / 30$ & 2 \\
\hline 10 & Cornered & $3 \cdot 3$ & $30 / 30$ & 2 \\
\hline 11 & Cornered & $2 \cdot 7$ & $60 / 30$ & 2 \\
\hline 12 & Cornered & $2 \cdot 7$ & $30 / 30$ & 2 \\
\hline
\end{tabular}

geometric and visual properties etc.) was created by transferring satellite images of the campus to the drawing program, with further measurements taken on-site. The layout of the test road, road markings and lane lines, footpaths, signs and trees alongside were modelled in three dimensions to mimic the real situation as closely as possible. The virtual chicanes were carefully placed on the most convenient locations on the test road and the drawings were then transferred to a three-dimensional design program. Additional elements such as surrounding buildings on the campus were photographed on-site and added to the lateral panels in this design package. All the drawings of the test road were then tiled together to complete the virtual environment where the driving tests would be carried out in the simulator. The simulator tests were carried out inside a real-size vehicle cabin with front, back, right and left simulator screens, as shown in Figure 1. In all the experiments, the weather conditions were chosen and modelled as sunny and the traffic volumes were deliberately set to free-flow conditions, as is the case most of the time on campus.

Before starting the tests, all drivers drove the simulator on a $1 \mathrm{~km}$ length of road to gain experience with the system. This

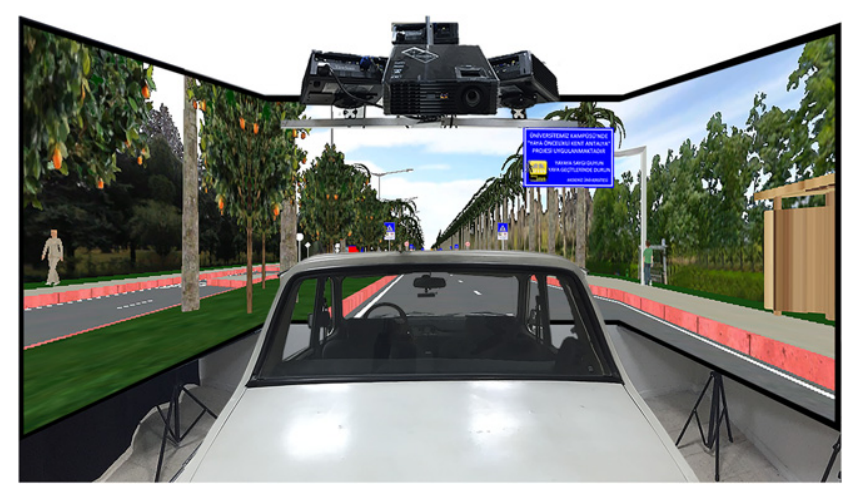

Figure 1. The simulator environment where the driving tests were performed training test gave them the opportunity to become familiar with the simulator's gearshift, steering wheel and brake systems. After training, the drivers had driven the simulator for only one lap along the $3 \mathrm{~km}$ route. During the real test, the route on which drivers would pass all the chicanes at least once was identified, and then each driver performed a $3 \mathrm{~km}$ drive, which took about 5-10 $\mathrm{min}$. At the end of the drive, the system created an individual data file for each driver. These data include about 80 positions per second and the spot speed $(\mathrm{km} / \mathrm{h})$ information with respect to that position.

The driving tests were randomised without gender discrimination. The gender distribution of the participants was selected to reflect the licensed female and male driver ratio in Turkey according to 2017 statistics from the Turkish Statistical Institute (TSI, 2017). Thus, of the 125 drivers in the simulator study, $76 \%$ were male and $24 \%$ were female. Based on a quick pre-test questionnaire survey, $79 \%$ were active drivers, $26 \%$ were inexperienced, 37\% were of intermediate level and $37 \%$ were professional drivers. In addition, $40 \%$ stated they drove calmly, $49 \%$ moderately and $11 \%$ aggressively. In terms of education level, $10 \%$ of the participants had an associate education level (high school diploma or equivalent), 55\% were of graduate level and 35\% were of postgraduate level.

\subsection{Vehicular trajectories}

Wheel track positioning rather than the centrelines of vehicles was employed during the tests for better accuracy and clarity in the analyses. The position data were therefore converted into the coordinates of the wheel paths. The amount of shift in the $x$ - and $y$-directions from the projection of the centreline was determined accordingly. This posed a new problem in that, according to the direction of the vehicle on the coordinate plane, there were shifts at different values in the $x$ - and $y$-directions at each point. Nevertheless, the different amounts of shifts performed in the $x$-and $y$-directions will always give the point at the same perpendicular distance from the projection of the centre of gravity. Figure 2 provides a better understanding. 

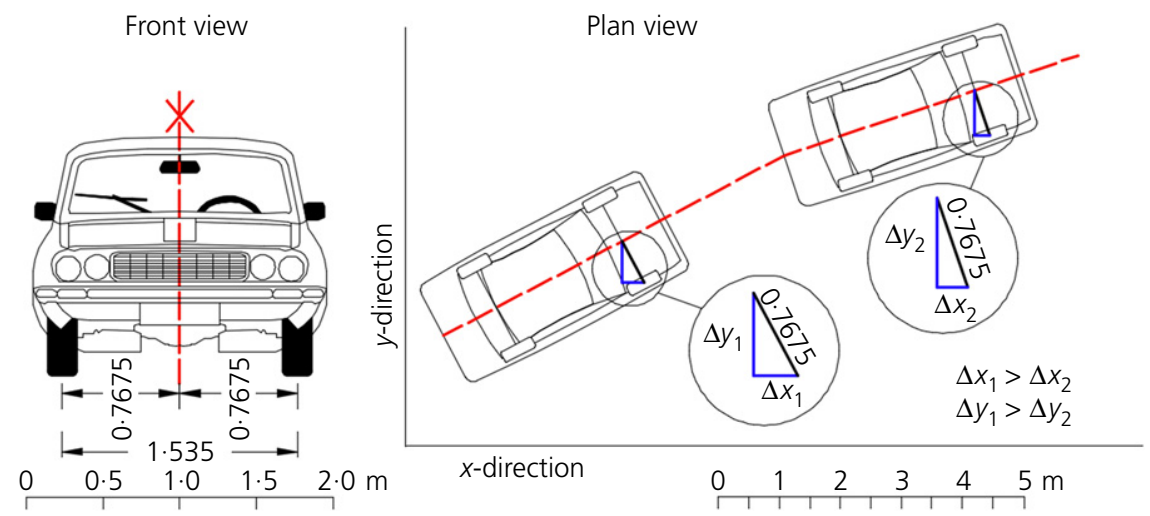

Figure 2. Horizontal distance between the projection of the centreline of the vehicle and the wheels (dimensions in $\mathrm{m}$ )

As also shown in the plan view in Figure 2, different amounts of shifts for the two different directions occurred. This problem was tackled by developing a piece of software in Matlab and the amount of shift for each point was computed by means of Equations 1-3 and the four steps listed in the following.

1. $m=\frac{y_{i+1}-y_{i}}{x_{i+1}-x_{i}}$

2. $\Delta x=0.7675 \sin \left[\tan ^{-1}(m)\right]$

\section{3. $\Delta y=0.7675 \cos \left[\tan ^{-1}(m)\right]$}

In Equations $1-3, y_{i}$, is coordinate $y$ of the $i$ th point, $x_{i}$ is coordinate $x$ of the $i$ th point, $m$ is the slope, $\Delta x$ is the amount of shift in the $x$-direction, $\Delta y$ is the amount of shift in the $y$-direction and 0.7675 (in $\mathrm{m}$ ) is the horizontal distance between the centreline of the vehicle and the wheel.

Equations 4-7 were then used to calculate the coordinates of the wheels for a point whose coordinates for the centreline were $O\left(x_{i}, y_{i}\right)$.

- Step 1 - for the left wheel.

If $O\left(x_{i+1}\right)>O\left(x_{i}\right)$ and the vehicle moves in the forward direction or if $O\left(x_{i+1}\right)<O\left(x_{i}\right)$ and the vehicle moves in the reverse direction.

- Step 2 - for the right wheel.

If $O\left(x_{i+1}\right)<O\left(x_{i}\right)$ and the vehicle moves in the forward direction or if $O\left(x_{i+1}\right)>O\left(x_{i}\right)$ and the vehicle moves in the reverse direction.

4. $\quad \operatorname{Right}\left(x_{i}\right)=\operatorname{Left}\left(x_{i}\right)=O\left(x_{i}\right)+\Delta x$
5. $\quad \operatorname{Right}\left(y_{i}\right)=\operatorname{Left}\left(y_{i}\right)=O\left(y_{i}\right)-\Delta y$

- Step 3 - for the left wheel.

If $O\left(x_{i+1}\right)<O\left(x_{i}\right)$ and the vehicle moves in the forward direction or if $O\left(x_{i+1}\right)>O\left(x_{i}\right)$ and the vehicle moves in the reverse direction

- Step 4 - for the right wheel.

If $O\left(x_{i+1}\right)>O\left(x_{i}\right)$ and the vehicle moves in the forward direction or if $O\left(x_{i+1}\right)<O\left(x_{i}\right)$ and the vehicle moves in the reverse direction.

6. $\quad \operatorname{Right}\left(x_{i}\right)=\operatorname{Left}\left(x_{i}\right)=O\left(x_{i}\right)-\Delta x$

7. $\operatorname{Right}\left(y_{i}\right)=\operatorname{Left}\left(y_{i}\right)=O\left(y_{i}\right)+\Delta y$

After the coordinate points providing the wheel tracks of each drive were obtained, they were fed into the previously designed test road in a drawing package and the points were joined by means of polylines. Since this manual operation is cumbersome, VisualLisp program codes that allowed drawing in both file formats were created with Matlab using these coordinate points. In this way, the wheel tracks of all drives were made automatically addible to the test road in a drawing program file and the wheel tracks of the drives performed by 125 people were visualised in this package. As an example, the trajectories of the wheel tracks in chicane design 3 on the two-lane dual carriageway are provided in Figure 3.

\subsection{Density maps}

The wheel tracks created in the drawing program were transferred to the ArcGIS analysis package and converted into density rasters. In this way, densely used areas due to the chicane applications were identified and the effects of different types of chicane geometry on the vehicular positions were analysed using the line density method. 


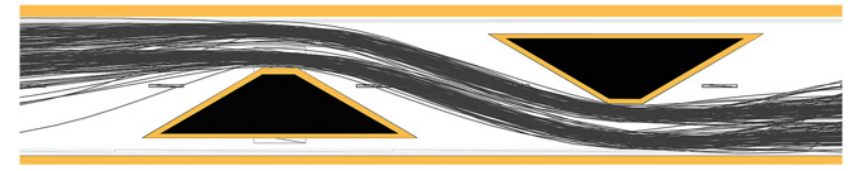

Figure 3. Trajectories of wheel tracks in chicane design 3; the direction of flow is from left to right

Data on positions and vehicle speeds were used to examine the speed densities in these areas. These data were defined as point data and the speeds were weighted using the kernel density method. In this way, the effects of the chicanes with different geometric characteristics on vehicles' speed change were examined. This operation was individually carried out specifically for each chicane zone. Finally, another analysis whereby all chicane zones were simultaneously evaluated was made using the same method. This allowed the detection of chicanes promoting the lowest speeds.

\subsection{Statistical analysis}

To ascertain if there was a significant difference among the average spot speeds occurring inside the chicanes, statistical analysis was carried out by evaluating the density rasters created for the speeds. As the number of chicanes was greater than two and since the only variable was speed, the decision was made to perform an analysis of variance. For this purpose, it was first checked whether or not the data fitted a normal distribution. According to the Kolmogorov-Smirnov test, it was found that $p=0 \cdot 000$, which showed that the data did not fit a normal distribution. Hence, the Kruskal-Wallis method was applied and it was found that $\chi^{2}=392.78$ and $p=0.000<0.05$. In this way, hypothesis $\mathrm{H}_{0}$ (i.e. there was no significant difference among the averages of the speeds in the different chicanes) was rejected. As a result of this test, the distribution of the speeds driven inside the chicanes is presented in Figure 4.

The speeds of the 125 drivers were examined separately for each chicane. The results showed that vehicle speeds were homogeneously distributed and the variances were equal. Thus, Dunnet's T3 post-hoc multiple comparison test was performed (for $<0.05$ significance level) to examine whether there was a significant difference between the average speeds of vehicles inside the 12 chicanes (Table 2 and Figure 5).

As shown in Table 2, the 12 chicanes were examined with Dunnet's T3 test and they were divided into dual combinations. A two-sample $t$-test was also performed to analyse vehicle speeds. These analyses showed that there was a

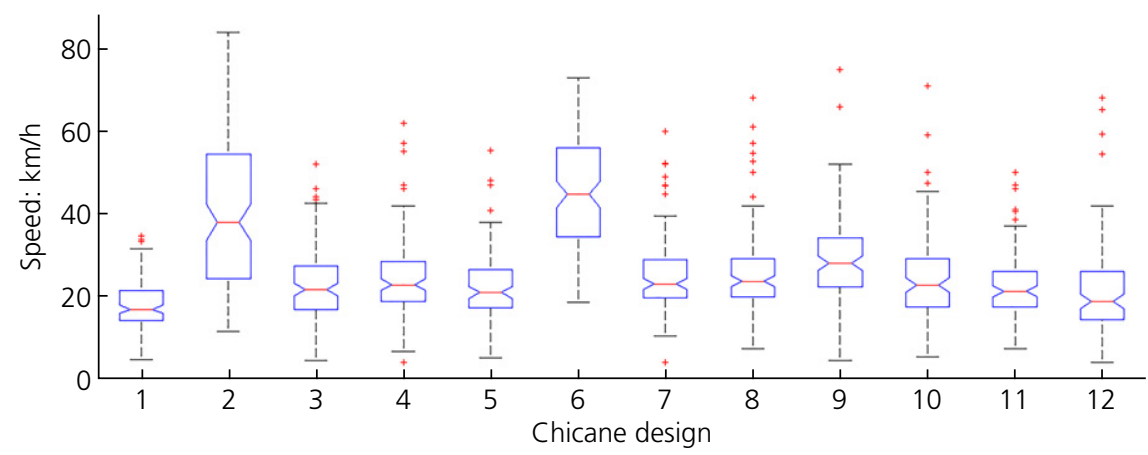

Figure 4. Distribution of speeds as a result of the Kruskal-Wallis test

Table 2. Dunnet's T3 multiple comparison for all chicanes (S means significant, NS means non-significant)

\begin{tabular}{|c|c|c|c|c|c|c|c|c|c|c|c|c|}
\hline Chicane design & 1 & 2 & 3 & 4 & 5 & 6 & 7 & 8 & 9 & 10 & 11 & 12 \\
\hline 1 & & $S$ & $S$ & $S$ & $S$ & $S$ & $S$ & $S$ & $S$ & $S$ & $S$ & $S$ \\
\hline 2 & $S$ & & $S$ & $S$ & $S$ & $S$ & $S$ & $S$ & $S$ & $S$ & $S$ & $S$ \\
\hline 3 & $S$ & $S$ & & NS & NS & $S$ & $S$ & $S$ & $S$ & NS & NS & $S$ \\
\hline 4 & $S$ & $S$ & NS & & NS & $S$ & NS & NS & $S$ & NS & $S$ & $S$ \\
\hline 5 & $S$ & $S$ & NS & NS & & $S$ & $S$ & $S$ & $S$ & NS & NS & $S$ \\
\hline 6 & $S$ & $S$ & $S$ & $S$ & $S$ & & $S$ & $S$ & $S$ & $S$ & $S$ & $S$ \\
\hline 7 & $S$ & $S$ & $S$ & NS & $S$ & $S$ & & NS & $S$ & NS & $S$ & $S$ \\
\hline 8 & $S$ & $S$ & $S$ & NS & $S$ & $S$ & NS & & $S$ & NS & $S$ & $S$ \\
\hline 9 & $S$ & $S$ & $S$ & $S$ & $S$ & $S$ & $S$ & $S$ & & $S$ & $S$ & $S$ \\
\hline 10 & $S$ & $S$ & NS & NS & NS & $S$ & NS & NS & $S$ & & NS & $S$ \\
\hline 11 & $S$ & $S$ & NS & $S$ & NS & $S$ & $S$ & $S$ & $S$ & NS & & $S$ \\
\hline 12 & $S$ & $S$ & $S$ & $S$ & $S$ & $S$ & $S$ & $S$ & $S$ & $S$ & $S$ & \\
\hline
\end{tabular}




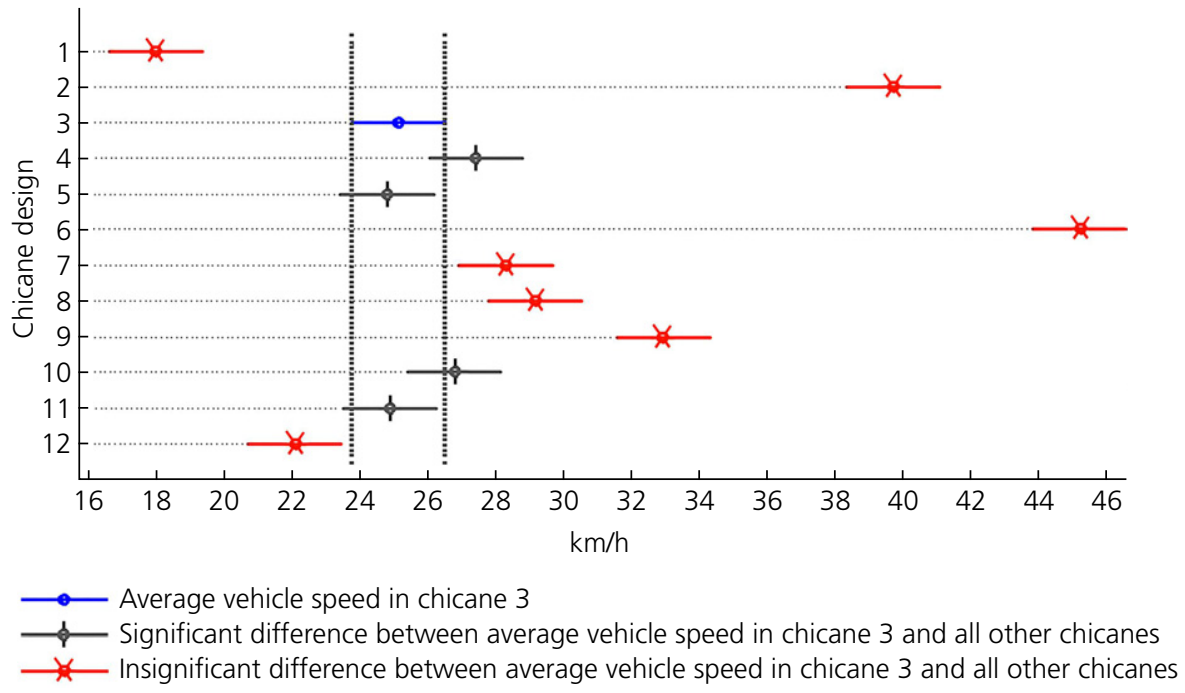

Figure 5. An example of Dunnet's T3 multiple comparison for chicane 3

significant difference between the dual chicanes. The results in Table 2 show that chicane designs 1, 2, 6, 9 and 12 had significant differences from the averages of the spot speeds $(\mathrm{km} / \mathrm{h})$ performed in all other chicanes. It can be seen from Figure 5 that there was a significant difference between the average vehicle speeds in chicane 3 and in all the other chicanes. While there was no significant difference between chicane 3 and chicanes $4,5,10$ and 11 , it was discovered that there was a significant difference between chicane 3 and chicanes $1,2,6,7,8,9$ and 12 .

\section{Findings}

First of all, how the different chicane geometric characteristics affected the utilisation of lanes was evaluated by examining the trajectories in the chicane zones. The trajectory densities for the two-lane dual carriageways are provided in Figure 6. A shading scale of light to dark was applied, with dark shading representing the areas with the most dense wheel tracks per unit area and light shading representing the areas with the least dense wheel tracks per unit area. Each chicane zone was evaluated within itself, meaning that the same colours in different chicane zones may not represent the same density values.

It can be seen from Figure 6 that, for the dual carriageways, chicane 1 caused a problem of significant disobedience to lane discipline both before and after the chicane, whereas chicane 6 did so before the chicane. As chicane 6 led to different levels of lateral shift in each vehicle, the wheel tracks did not occur individually, but a situation as if there had been a single track occurred. This was because chicane 6 had an effective width greater than that of the other chicane designs. On the other hand, no significant lack of lane discipline was seen in chicanes 2, 3, 4, 5, 7 and 8 . The chicane in which lane discipline was most obeyed was chicane 2 , with an effective width of $2.7 \mathrm{~m}$. In chicane 7 , a forced left turn due to the driving track was present in the last section. A deviation from the travel path (at the far right end of the road) does not have anything to do with the chicane in question. It is due to a left turn on the road.

Examining the designs for the two-lane single carriageways (Figure 7) it is seen that, before chicanes 9 and 10, the trajectories shifted towards the lane line slightly more when compared with chicanes 11 and 12. This indicates that there was an increase in obeying lane discipline, again depending on the decrease in the effective width of the chicane. It cannot be interpreted that lane discipline was not obeyed in any of these designs.

In Figures 6 and 7, created for the trajectory densities, both situations in which the right and left wheel tracks clearly appeared are seen and sometimes a single wheel track becomes evident. This is because the vehicles were in different lateral positions at the same point. Sometimes, a vehicle may pass a point with its left wheel and another vehicle may pass over the same point with its right wheel. This increases the density at that point, thereby creating a view as if there was a single wheel track.

The performances of the chicanes in terms of speed reduction were evaluated by examining the speed densities in the chicane zones (Figures 8 and 9). In the figures, generated by weighting according to the speeds, the light-shaded areas denote the maximum speed and the minimum density, while the darkshaded areas indicate the minimum speed and the maximum density. The term 'density' here refers to the number of wheels passing per unit area. The change in speed in each chicane zone was rated within itself, and so the same shades in the different chicanes do not represent the same speeds. In these figures, the most ideal situation for a chicane is a concentration 


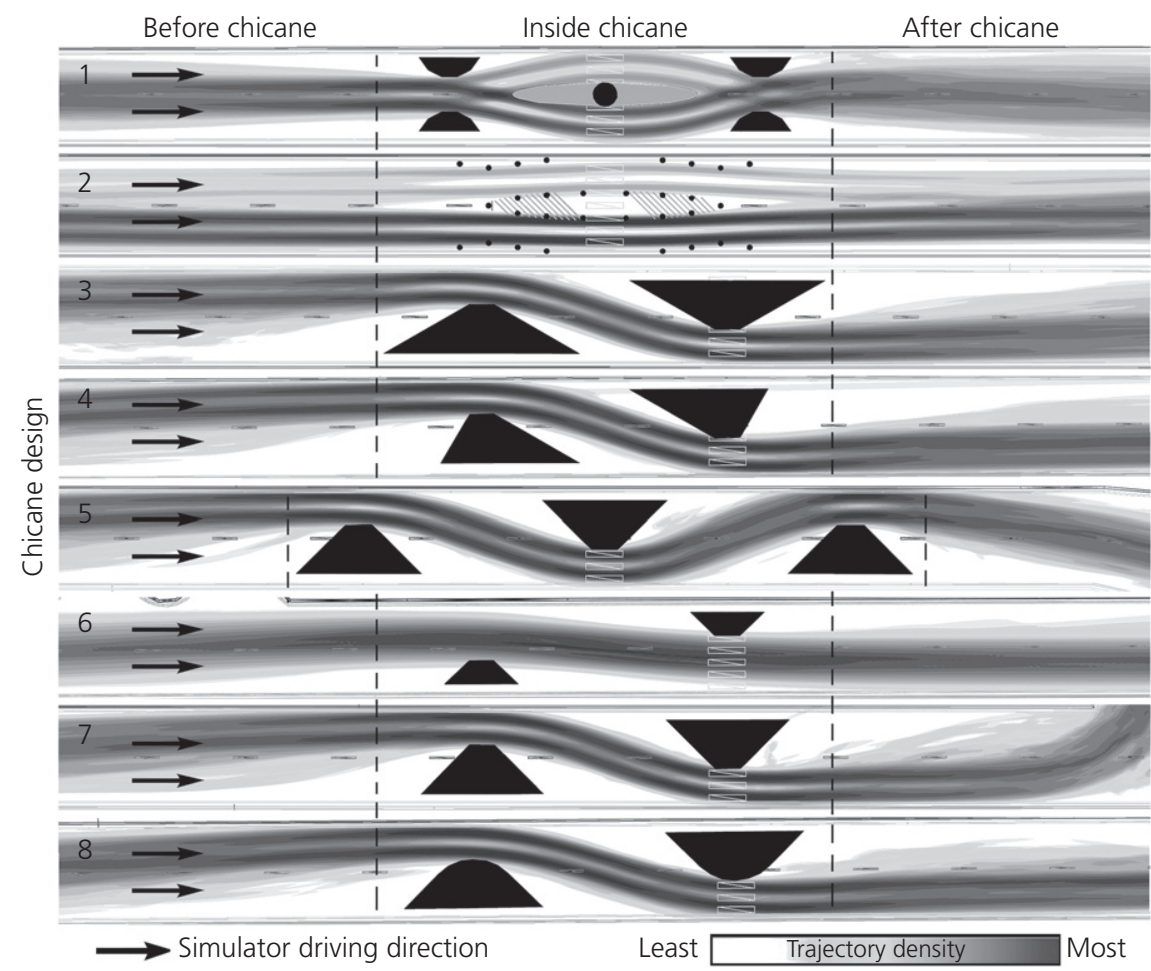

Figure 6. Trajectory densities of chicanes 1-8

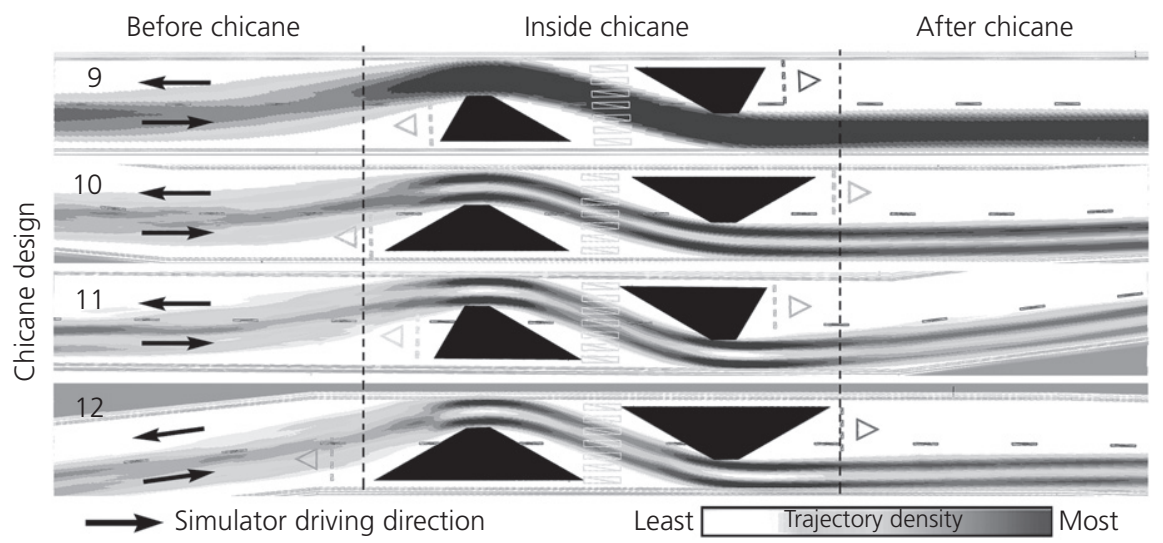

Figure 7. Trajectory densities of chicanes 9-12

of light shades (maximum speed) before the chicane and dark shades (minimum speed) inside the chicane.

Figure 8, for the two-lane dual carriageways, shows that dark shades were dominant before and inside chicanes 2 and 6 , meaning that these chicanes failed to reduce vehicle speeds. The reason for this failure is that these chicanes created a deflection at a level at which they could be passed using very small manoeuvres. The reduction of the effective width to $2.7 \mathrm{~m}$ in chicane 2 failed to produce a reduction in speed and the effective width of $5 \mathrm{~m}$ in chicane 6 had the same effect (i.e. vehicles moved at the same speed before and inside the chicane). This was not the case for the other chicanes. Hence, besides the effective width, the widths of the chicane islands and their features for deflecting vehicles are also important regarding speed reduction. 
Transport

Volume 175 Issue 4
Geometric optimisation of chicanes using

driving simulator trajectory data

Akgol, Gunay and Aydin

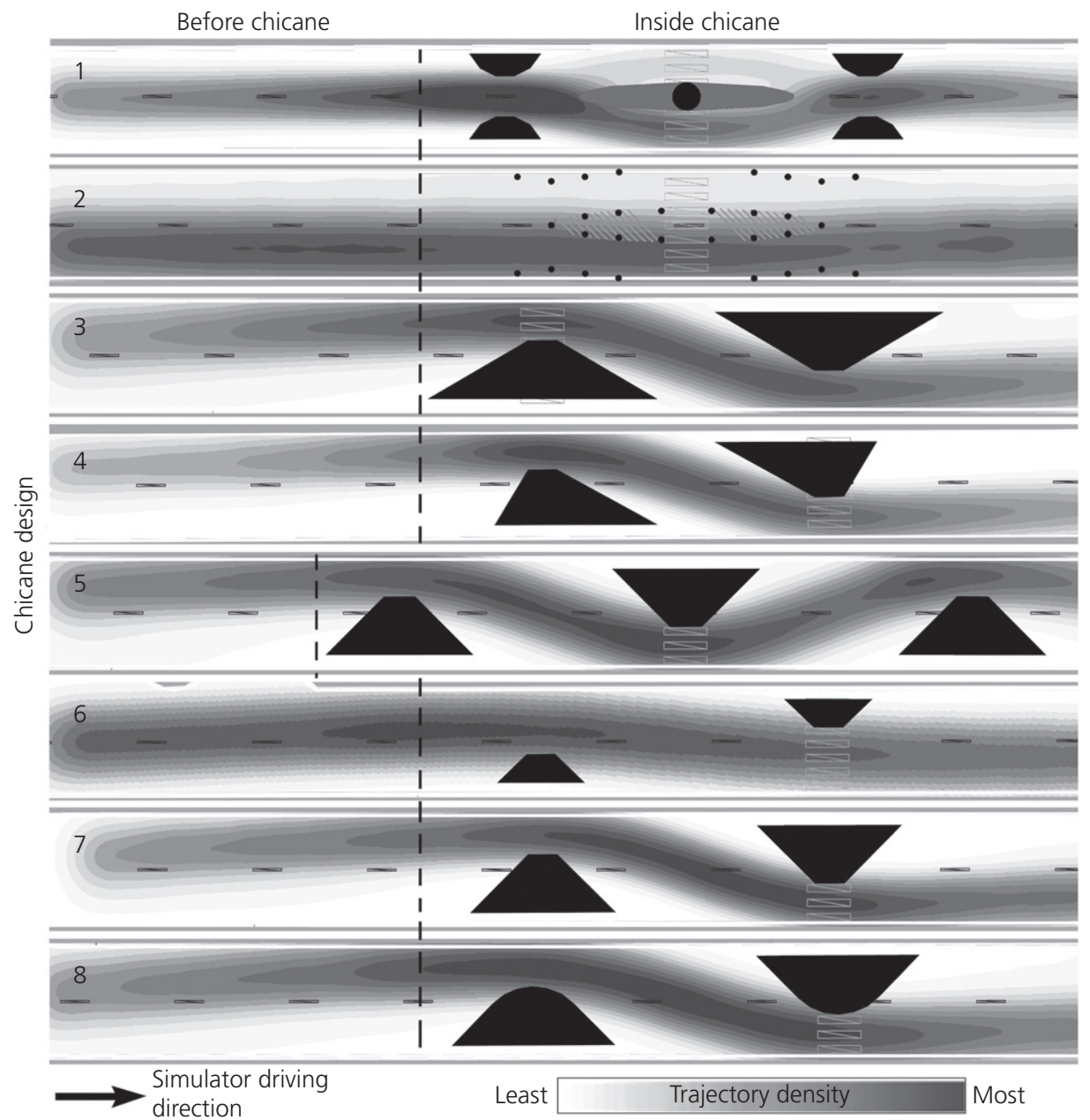

Figure 8. Speed densities of chicanes 1-8

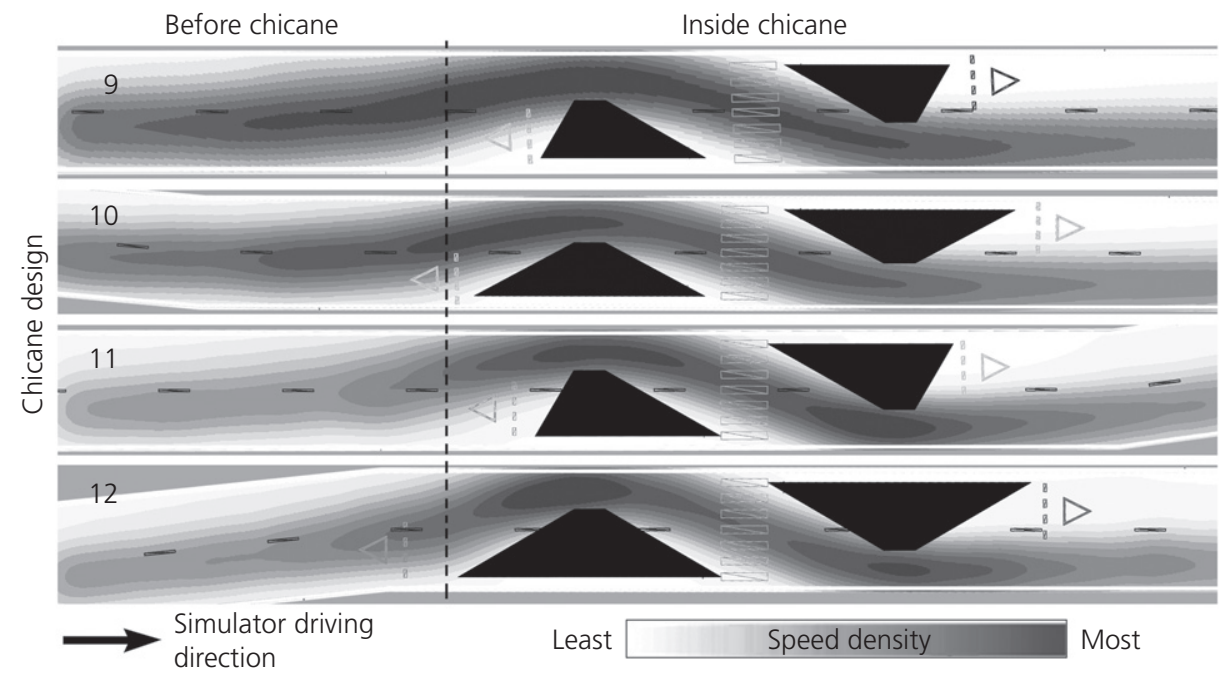

Figure 9. Speed densities of chicanes 9-12 
Table 3. Preferred and non-preferred chicanes

\begin{tabular}{|llll|}
$\begin{array}{l}\text { Chicane } \\
\text { design }\end{array}$ & Trajectory & Speed density & $\begin{array}{l}\text { Trajectory and } \\
\text { speed density }\end{array}$ \\
\hline 1 & Not preferred & Preferred & Not preferred \\
2 & Preferred & Not preferred & $\begin{array}{l}\text { Not preferred } \\
\text { Preferred }\end{array}$ \\
3 & Preferred & Preferred & Preferred \\
4 & Preferred & Preferred & Preferred \\
5 & Preferred & Preferred & Pred \\
6 & Not preferred & Not preferred & Not preferred \\
7 & Preferred & Preferred & Preferred \\
8 & Preferred & Preferred & Preferred \\
9 & Preferred & Not preferred & Not preferred \\
10 & Preferred & Not preferred & Not preferred \\
11 & Preferred & Preferred & Preferred \\
12 & Preferred & Preferred & Preferred \\
\hline
\end{tabular}

Examination of Figure 9 for the two-lane single carriageways reveals that chicanes 9 and 10 failed to reduce speeds because the effective widths of these chicanes was $3 \cdot 3 \mathrm{~m}$. No such problem was encountered in chicanes 11 and 12, with effective widths of $2.7 \mathrm{~m}$.

From these results, the preferred and non-preferred chicanes in terms of trajectories and speed densities were determined and the results are shown in Table 3. Figure 10 was created in order to compare the efficiency of the preferred chicanes in reducing the vehicle speeds.
The efficiency of the preferred chicane designs in terms of speed reduction is shown in descending order in Figure 10. Darkening of the shades in the figure represents an increase in the efficiency in speed reduction. Of the seven preferred designs, chicane 12 turned out to be the most efficient design with respect to speed reduction, while chicane 8 was the most inefficient. Chicanes 11 and 12 were found to be more efficient in speed reduction than the others since the effective width for vehicles in these chicanes was $2.7 \mathrm{~m}$. Chicane 5 was efficient in speed reduction as it is a three-island design. In addition, the leading angle of chicane 12 was $30^{\circ}$, which played a role in the fact that it was more efficient in speed reduction than the other designs.

In this comparison of 12 different chicanes, evaluations were made with respect to the design characteristics of the chicanes according to the position and speed data of vehicles passing through them. The lateral positions of the vehicles were examined according to the trajectories generated and it was concluded that chicanes 1 and 6 led to a lack of lane discipline. This demonstrates that the entrance width of a chicane should definitely not be at the centre (as in chicane 1) and that the effective width should not be greater than the lane width (as in chicane 6). Looking at the speed densities in the chicanes, there was hardly any speed reduction in chicane 6 due to the effective width. On the other hand, in chicane 2 , since the drivers were able to pass using a small manoeuvre within the same lane even though the effective width was reduced

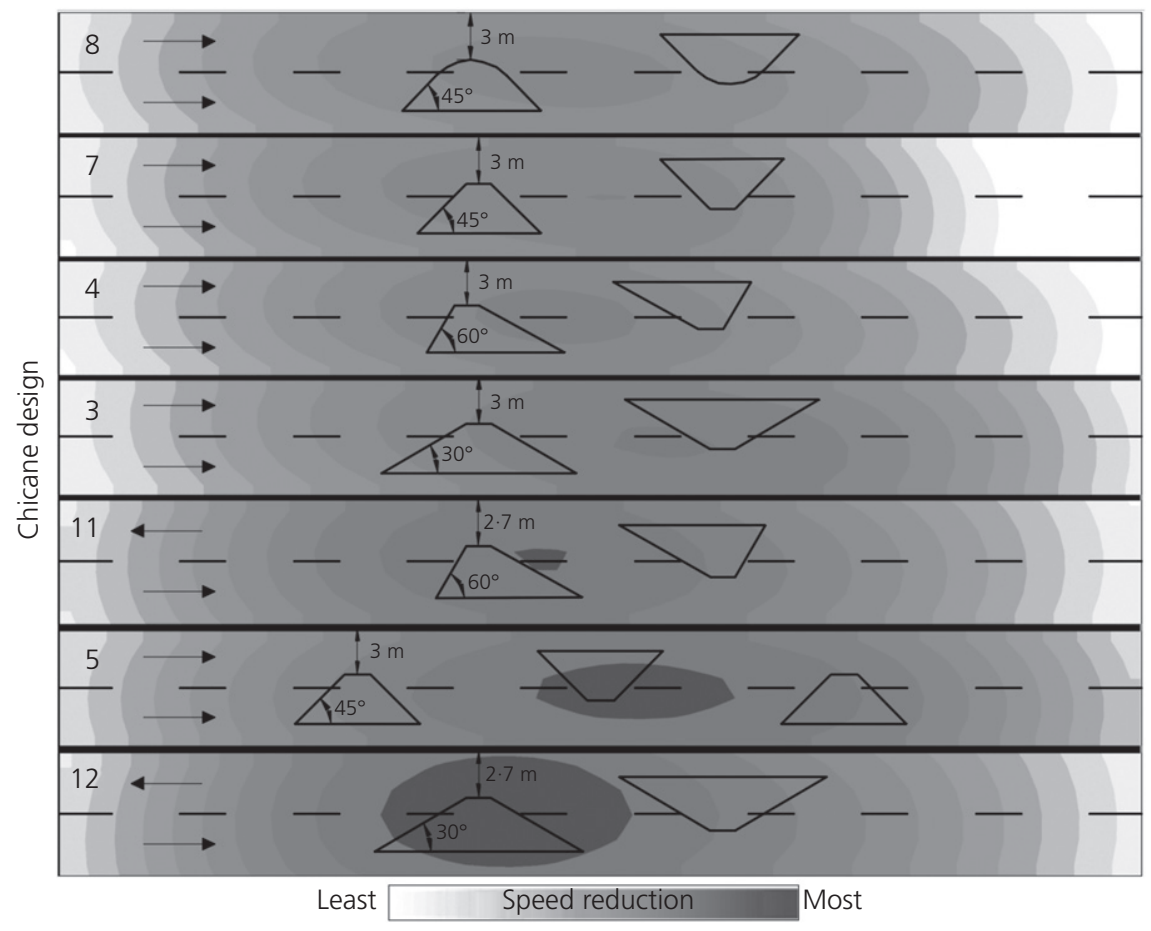

Figure 10. Comparison of the efficiency of chicanes $3,4,5,7,8,11$ and 12 in speed reduction 
to $2.7 \mathrm{~m}$, this design was not successful in reducing the speed either. Hence, to reduce the speed in chicane applications, a vehicle should be enabled to change lane completely. For an effective width of $3.3 \mathrm{~m}$, even though the possibility of a complete lane change was provided while the vehicle was passing through the chicane, chicanes 9 and 10 showed rather reduced efficiencies in speed reduction. Examination of the other designs revealed that setting the effective width at $3 \mathrm{~m}$ or less will be useful in reducing speeds in chicanes. Comparing the chicane islands of chicane 7 (cornered) and chicane 8 (parabolic), the cornered design was slightly more efficient in speed reduction. Nevertheless, since the difference was insignificant, it cannot be stated that this characteristic is directly effective (see Table 2). Regarding the leading angles of the chicane islands, it was found that designs with a leading angle of $30^{\circ}$ were evidently more successful than other designs with the same effective width. Comparing the chicane designs with two or three islands, chicane 5 (with three islands) performed better than all the other chicanes in terms of speed reduction, except for chicane 12. However, the fact that chicane 12, with two islands, turned out to be the most efficient design for speed reduction decreases the potential of three-island chicanes being preferred in terms of economy. Hence, choosing chicane designs with an effective width of $3 \mathrm{~m}$ or less, a leading angle of $30^{\circ}$ and two islands will yield more productive results. Among the considered designs, chicanes 3 and 12 were the most efficient.

\section{Conclusion}

Chicanes with different designs were compared by examining driver behaviours with the intention of determining the optimum design characteristics. A methodology that provided an opportunity for a detailed examination of vehicular movements was first developed. This examination was carried out using VisualLisp programming, which provided the automatic transfer of vehicular trajectories to a commercial drawing package. By transferring the obtained data to an ArcGIS analysis tool, the speeds of the drivers were visualised depending on their position data. Drives in the simulator that showed behaviour that would not reflect reality (as the density methods of the ArcGIS package were used) were automatically eliminated and more accurate results reflecting the general state were thus obtained. Even though the position data were weighted only according to the speed data in this study, varying driver behaviours could also be examined using the same method in future work.

The results of this study showed that the design characteristics of chicanes 3 and 12 gave the most successful performances in terms of lane discipline and speed reduction. In addition to the characteristics of these chicanes, consideration of vehicular movements indicated that the choice of parabolic rather than cornered chicane islands may enable drivers to perform a safer drive. Examination of the drives inside the chicanes showed that the drivers preferred to take the shortest path in order to exit the chicane as soon as possible instead of returning to their own lane after they had passed the first island. This meant that two vehicles facing each other failed to pass the chicane simultaneously. Hence, by placing a small channelling system in the central area between two islands, it may be ensured that two vehicles can pass the chicane simultaneously. Finally, the dead areas immediately around the chicane islands should be of particular interest. Including these areas within the chicane islands and reducing the leading/trailing face angles of the islands is considered to be ongoing work.

The results of this study should be helpful to transportation authorities in the design of suitable and safer chicanes to increase road safety on urban minor roads. In future studies, various limitations of this work need to be tackled. For example, investigation of many more different chicane types and urban minor road characteristics may provide better understanding to achieve optimum chicane geometry.

\section{Acknowledgement}

The authors thank the Coordination Unit for Scientific Research Projects at Akdeniz University for supporting this study (project no. FBA-2015-225).

\section{REFERENCES}

Bahar GB (2007) Guidelines for the Design and Application of Speed Humps. Institute of Transportation Engineers, Washington, DC, USA.

Bayer F and Hauser J (2012) Trajectory optimization for vehicles in a constrained environment. In Proceedings of the 51st IEEE Conference on Decision and Control. Institute of Electrical and Electronics Engineers, Piscataway, NJ, USA, pp. 5625-5630.

Bergeron J and Paquette M (2014) Relationships between frequency of driving under the influence of cannabis, self-reported reckless driving and risk-taking behavior observed in a driving simulator. Journal of Safety Research 49: 19-24.

Calvi A and De Blasiis MR (2011) Driver behavior on acceleration lanes driving simulator study. Transportation Research Record 2248: 96-103.

Calvi A, Benedetto A and De Blasiis M (2012) A driving simulator study of driver performance on deceleration lanes. Accident Analysis \& Prevention 45: 195-203.

Corkle J, Giese JL and Marti MM (2001) Investigating the Effectiveness of Traffic Calming Strategies on Driver Behavior, Traffic Flow and Speed. Minnesota Local Road Research Board, St. Paul, MN, USA.

DfT (Department for Transport) (2006) General Principles of Traffic Control by Light Signals. DfT, London, UK, Traffic Advisory Leaflet $1 / 06$.

Drăgoi MV and Navalušić S (2013) Converting point cloud to revolved solids. Applied Mechanics and Materials 371: 468-472.

Duivenvoorden K, Hogema J, Hagenzieker M and Wegman F (2015) The effects of cyclists present at rural intersections on speed behavior and workload of car drivers: a driving simulator study. Traffic Injury Prevention 16(3): 254-259.

Filip AC and Neagoe I (2010) Simulation of the metal spinning process by multi-pass path using AutoCAD/VisualLISP. In Latest Trends on Engineering Mechanics, Structures, Engineering Geology (Martin O and Zheng X (eds)). WSEAS Press, Corfu Island, Greece, pp. 161-165. 
Gelau C, Sirek J and Dahmen-Zirnmer K (2011) Effects of time pressure on left-turn decisions of elderly drivers in a fixed-base driving simulator. Transportation Research Part F: Traffic Psychology and Behaviour 14(1): 76-86.

Gunay B and Woodward D (2007) Lateral position of traffic negotiating horizontal bends. Proceedings of the Institution of Civil Engineers - Transport 160(1): 1-11, https://doi.org/10.1680/ tran.2007.160.1.1.

Hu L and Pei Y (2012) Analysis of driver's physiological characteristics under ice-snow conditions based on driving simulator. In Emerging Systems for Materials, Mechanics and Manufacturing (Zhang YP, Zhou LH and Mao E (eds)). Trans Tech Publications, Zurich, Switzerland, pp. 547-550.

Kanagaraj V, Asaithambi G, Toledo T and Lee TC (2015) Trajectory data and flow characteristics of mixed traffic. Transportation Research Record 2491: 1-11.

Kim SM, Lee SK, Lee TK, Lee SB and Kim BM (2011) Process design program for multistage profile drawing from round material. Journal of the Korean Society for Precision Engineering 28(3): 377-382.

Lee SK, Lee SB and Kim BM (2010) Development of intermediate die shape design program for multi-pass shape drawing by using VisualLISP. Transactions of Materials Processing 19(4): 242-247.

Lim JB, Hong JY, Lee SB, Jung SH and Hwang JR (2010) A study on driver's psychological responses to VMS traffic information using driving simulator. Journal of The Korea Institute of Intelligent Transport Systems 9(3): 20-29.

Macbeth AG (1988) Calming arterials in Toronto. In Proceedings of the 68th Annual Meeting of the Institution of Transportation Engineers. Institution of Transportation Engineers, Washington, DC, USA, CD-ROM.
Nygardhs S, Lundkvist SO, Andersson J and Dahlback N (2014) The effect of different delineator post configurations on driver speed in night-time traffic: a driving simulator study. Accident Analysis \& Prevention 72: 341-350.

Park JD, Kim SY, Han MS and Jeon EC (2007) Application of automatic design program for aspheric lens design. Journal of the Korean Society of Manufacturing Process Engineers 6(3): 3-8.

Pecher C, Lemercier C and Cellier JM (2012) Effects of sadness on drivers' behaviour: an empirical study using emotional induction and a driving simulator. In Advances in Traffic Psychology (Sullman M and Dorn L (eds)). Ashgate Publishing, Farnham, UK, pp. 43-50.

Peterson ER (2006) The Effectiveness of Low-cost Traffic Calming Applications Appropriate for Main Streets Through Rural Communities. Master's thesis, Iowa State University, Ames, IA, USA.

Rumschlag G, Palumbo T, Martin A et al. (2015) The effects of texting on driving performance in a driving simulator: the influence of driver age. Accident Analysis \& Prevention 74: 145-149.

Sayer I, Parry D and Barker J (1998) Traffic Calming - An Assessment of Selected on-Road Chicane Schemes. Transport Research Laboratory, Crowthorne, UK, Report 313.

TSI (Turkish Statistical Institute) (2017) Road Traffic Accident Statistics. TSI, Ankara, Turkey.

Vollrath M, Schleicher S and Gelau C (2011) The influence of cruise control and adaptive cruise control on driving behaviour - a driving simulator study. Accident Analysis \& Prevention 43(3): 1134-1139.

Yang Q, Overton R, Han LD, Yan X and Richards SH (2014) Driver behaviours on rural highways with and without curbs - a driving simulator based study. International Journal of Injury Control and Safety Promotion 21(2): 115-126.

\section{How can you contribute?}

To discuss this paper, please email up to 500 words to the editor at journals@ice.org.uk. Your contribution will be forwarded to the author(s) for a reply and, if considered appropriate by the editorial board, it will be published as discussion in a future issue of the journal.

Proceedings journals rely entirely on contributions from the civil engineering profession (and allied disciplines). Information about how to submit your paper online is available at www.icevirtuallibrary.com/page/authors, where you will also find detailed author guidelines. 